Western University

Scholarship@Western

Biology Publications

Biology Department

Fall 10-2014

\title{
Editorial overview: Environmental physiology: Insect environmental physiology
}

Brent J. Sinclair

Western University, bsincla7@uwo.ca

Follow this and additional works at: https://ir.lib.uwo.ca/biologypub

Part of the Animal Sciences Commons, Biology Commons, Cell Biology Commons, Entomology Commons, and the Molecular Genetics Commons

Citation of this paper:

Brent J Sinclair, Editorial overview: Environmental physiology: Insect environmental physiology, Current Opinion in Insect Science, Volume 4, 2014, Pages ix-x, ISSN 2214-5745, http://dx.doi.org/10.1016/j.cois.2014.08.009. (http://www.sciencedirect.com/ science/article/pii/S2214574514000625) 
Editorial overview: Insect environmental physiology

Brent J. Sinclair

Department of Biology, University of Western Ontario, London, ON N6G 1L3, Canada

e-mail: $\underline{\text { bsincla7@@uwo.ca }}$

Insects dominate the diversity and function of terrestrial ecosystems, and to do so, they must overcome a range of environmental stressors. As ectotherms, they are subject to the biological effects of extreme temperatures; with a high surface area: volume ratio, they are subject to extremes of water loss; and with the exceptional efficiency of the tracheal system comes the potential for high levels of intracellular oxidative stress. Environmental physiology is the integrative discipline that cuts across molecular mechanisms, physiological function and organismal biology, and the diversity of insects allows tests of broader theory. In this issue of Current Opinion in Insect Science, I had the opportunity to commission articles that cut across insect environmental physiology.

The first two articles address two very different approaches to understanding the molecular underpinnings of insects' interactions to their environment.

First, Scott Hayward reviews the use and potential of functional 'omics' techniques for understanding insect responses to environmental stress, focusing on transcriptomics, proteomics, and metabolomics. Hayward identifies two particular weaknesses with omics work currently conducted in the context of environmental physiology - first, that the correspondence among types of omics is often not strong: few studies have identified close consistent processes in (for example) both the metabolome and the transcriptome. Second, although omics approaches are excellent for generating hypotheses about underlying processes that response to stress, few studies have followed those hypotheses through to establish causal mechanisms, but the emergence of the CRISPR/Cas9 system for gene editing (among other emerging technologies) may facilitate testing of hypotheses in non-model species in the near future. Hayward is also positive about the potential for other omics techniques, such as lipidomics, to provide a more targeted approach to identifying mechanisms. The success of omics in understanding insect stress responses will, however, not come just from advancements in technology, but also from refinement of experimental design. In particular, Hayward points out that significant gains may be made from designing experiments to dissect the temporal and tissue- and organelle- specific patterns in stress responses, treating them as the dynamic processes we know them to be.

Next up, Wheat and Hill provide an update on progress in understanding the role of the branch-point glycolysis enzyme phosphoglucose isomerase (PGI) in determining the physiological performance of insects. In contrast to the hypothesis-free approach afforded by transcriptomics, $P G I$ has been a candidate gene underlying variation in thermal tolerance for nearly forty years. Since then, variation in PGI has been associated with thermal tolerances in an array of insects, from beetles to bees, and Wheat 
and Hill collate the recent developments across the arthropod phylogeny. However, it is one thing to identify correlations and (in keeping with Hayward's observations) a completely different thing to identify causation and mechanisms; Wheat and Hill examine the two main schools of hypotheses for the association between PGI and performance. The first are 'flux hypotheses' that posit that PGI's role as a key branch-point enzyme allows it to regulate glycolytic flux, the other is the 'moonlighting hypothesis' that proposes epistatic effects of PGI enzymes are the cause of the observed associations. Wheat and Hill point out that a true understanding of the mechanisms underlying variation in metabolism (and the role of PGI in that variation) requires investigation at multiple levels of organisation - and ultimately manipulation of PGI enzyme abundance or function, and while variation in amino acid sequence and molecular analyses suggesting positive selection have been identified in a number of species, far fewer investigations have addressed the 'sharp end' of measuring biochemical function or organismal performance and fitness. Filling these gaps is clearly essential to link mechanism to performance and to resolve the PGI story.

The next two articles focus on insect gas exchange and metabolism. Contreras and colleagues provide an update on the myriad hypotheses explaining the prevalence and purpose of the widely observed discontinuous gas-exchange cycle (DGC). The striking DGC of intermittent 'breathing' by insects, has attracted attention because of its prevalence across arthropod groups, begging explanations for its evolutionary origins and current utility. This debate has often been contentious, and Contreras et al. review the many explanations, focusing on the group of recent, adaptive, hypotheses that posit the DGC evolved as a means for insects - which effectively 'mainline' oxygen through their efficient tracheal system - to regulate the delivery of oxygen to their cells. They contend that, whatever its present advantages, DGC originally evolved as a mechanism of respiratory regulation.

Harrison et al. take a step further back, and provide a demonstration of the utility of insects for testing wider theory, in this case the 'Metabolic Theory of Ecology' (MTE) explanation for the hypometric scaling of metabolic rate with body size. They lay out several of the current theories, but explicitly test the MTE theory. They present a brief meta-analysis of experiments that examine the effect of atmospheric oxygen manipulation (which therefore manipulates oxygen supply to the tissues) on metabolic performance in active and resting insects of a huge range of body sizes. They find little sizedependence of critical $\mathrm{PO}_{2}$ (the partial pressure of oxygen below which insect performance declines), which they interpret as a killer blow to the MTE - and suggesting that there is ample opportunity for the development and testing of new models for the mechanisms underlying the clearly-established relationships between body size and metabolic rate in insects.

Finally, the purpose of environmental physiology is to understand the physiology of insects in the context of their environment, and John Terblanche reviews the growing literature that seeks to understand the performance of insects in the field. Terblanche focuses on are those that examine the impacts of pre-conditioning of insects on their performance when released - particularly relevant for field release of sterilised pest, or fertile beneficial insects. This work draws on experiments both with species used in integrated pest management e.g. the codling moth, Cydia pomonella), and more theoretical studies that take advantage of the deep understanding of Drosophila melanogaster genetics and thermal biology. The difficulty of interpreting these studies - or predicting their outcomes - is 
underlined in a section about the links (and lack of links) between field and laboratory estimates of fitness. This is clearly a fertile field that promises to link the sometimes esoteric laboratory investigation of environmental physiology in the laboratory to real-world applicability.

What can we conclude from this somewhat eclectic overview of the breadth of insect environmental physiology? There are many more questions than answers in the articles in this section, but I think that it would be incorrect to conclude that environmental physiology has made little progress in understanding the interactions of insects with their environment, as topics I chose to cover are deliberately those in which there has been significant recent debate, and rapid progress. Indeed, much of that progress may be accelerated by the techniques discussed by Hayward, and see benefit through incorporation into applications that require insects to be more or less tolerant of their environment, as discussed by Terblanche. Perhaps this also reflects one of the specific challenges of insect environmental physiology in general - the enormous species diversity of insects is matched by a vast array of habitats, lifestyles and responses to environmental challenges. Environmental physiologists (and comparative physiologists in general) therefore walk a fine line between 'stamp collecting' and deep, mechanistic studies. The former are effectively physiological natural history, yet are essential for answering the broad, evolutionary questions posed by broad-scale evolutionary problems such as those addressed by Contreras et al. and Harrison et al., while the intensity and species-specificity of the deep mechanistic studies risk hampering their more general application. Thus, I think the key conclusion here is that there is plenty of room still for novel, deep, and broad investigations in insect environmental physiology, with a general application of predicting and managing insect performance. 


\section{Vita}

Brent Sinclair is an Associate Professor of Biology at Western University. Brent completed his undergraduate degree and PhD in Zoology at the University of Otago in New Zealand, where he worked on the thermal biology of alpine and Antarctic arthropods. After postdocs at the University of Stellenbosch, South Africa, and the University of Nevada, Las Vegas, he began at Western University in 2006. Brent's research is still focused on the cold tolerance of insects and other invertebrates, and he takes an integrative approach to (some would say he 'dabbles inveterately in') low temperature biology, ranging from single-gene studies through to continental-scale studies of winter ecology on a diversity of species ranging from Drosophila melanogaster to New Zealand stick insects. 